\title{
Note on Software III. Interpreter MACRO/2 and MACRO/3
}

\author{
Sigeru OKamoto* and Yoshiaki NaKamURA*
}

\begin{abstract}
MINI has no floating point arithmetic unit and we developed the interpreters MACRO/2 and MACRO/3, by which floating point arithmetic could be performed, for MINI. The determination of the effective address in the language interpreted by these interpreters is same as in ordinary machine language.

Using these interpreters, we can also calculate the values of the elementary functions such as sine, cosine etc. We have also the programs by which we can read and write the numbers with I, F or E type in MACRO/2 (or MACRO/3).
\end{abstract}

\section{Introduction}

A word in MINI is made up of 16 bits, and the machine language of MINI operates upon a 16-bits-word. Arithmetic operations operate only upon a 16bits-word, and only by software we can perform the floating point arithmetic. So we designed the interpreter $\mathrm{MACRO} / 2$ or $\mathrm{MACRO} / 3$ by which we could compute using data of the 2 (or 3 ) words of the floating point representation.

In this note we introduce principally $\mathrm{MACRO} / 2$, since $\mathrm{MACRO} / 3$ is programed very similar to MACRO/2.

\section{Format of data}

We represent a data $X$ in MACRO/2 by means of 32 bits of two consecutive words in which the mantissa is composed of 23 bits and the exponent 9 bits as shown in Fig. 1.

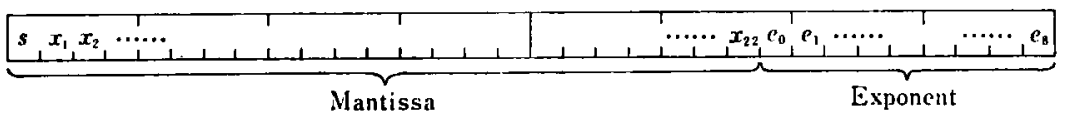

Fig. 1. Format of Data

If $X=0$, we put $s=x_{1}=\cdots=x_{22}=e_{0}=\cdots=e_{8}=0$. If $X \neq 0$, the mantissa

Received January 30, 1977.

* Department of Mathematics, Faculty of Science, Ibaraki University, Mito, Japan. 
represents a binary fraction

$$
f=(1-2 s) \sum_{i=1}^{22} x_{i} / 2^{i}
$$

where $s$ is the sign bit and $x_{1} \neq 0$ in general, and the exponent represents an integer

Then

$$
n=\sum_{i=1}^{7} 2^{i} e_{8-i}+2^{8}\left(e_{0}-1\right)
$$

$$
X=f \cdot 2^{n}
$$

For convenience' sake, some pseudo registers are prepared as follows:

1. The pseudo main register consisting of 4 words: MR

2. The pseudo sub-register consisting of 4 words: SR

3. The working register consisting of 2 words: WR

Moreover, we have the single word registers OP in which the operand of the pseudo instruction is stored and EA in which the effective address determined by the pseudo instruction is stored.

In MACRO/3, the mantissa part of the pseudo registers MR and SR are composed of 3 words ( 48 bits), and WR is also composed of 3 words.

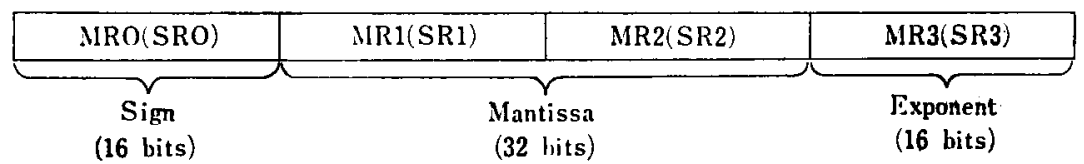

Fig. 2. Format of Registers MR and SR

\section{Pseudo instructions (Macro-instructions)}

A pseudo instruction interpreted by $\mathrm{MACRO} / 2$ is made of a single word, and the format of it except some is constructed as the memory reference instruction in MINI. It is shown in Fig. 3.

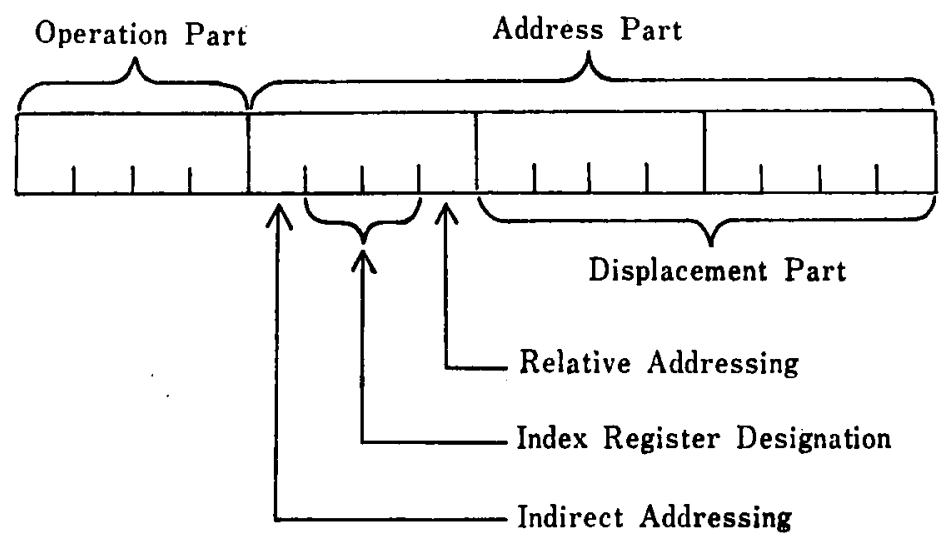

Fig. 3. Format of Pseudo Instructions 
Using the basic assembler $\mathrm{ON} / 1$, the pseudo instructions can be written by the corresponding mnemonic codes. The operator parts of them are shown in Table 1. The effective address is determined as in the "ordinary" instructions.

Particularly, when the special pseudo instructions in Table 2 are used, they are assembled into $8 \times \times \times$, and the subroutines prepared in MACRO/2 (or MACRO/3) are called in the execution of the program.

We have also the pseudo instructions for the elementary functions as in Table 3.

Table 1. Pseudo Instructions (Fundamentals)

\begin{tabular}{|c|c|c|}
\hline $\begin{array}{c}\text { Machine code } \\
\text { (Hexadecimal number) }\end{array}$ & $\begin{array}{l}\text { Mnemonic code } \\
\text { (in } \mathrm{ON} / 1 \text { ) }\end{array}$ & Operation \\
\hline $1 \times \times \times$ & $\mathbf{A} / \cdots$ & $(\mathrm{MR})+($ e. a.' $) \rightarrow \mathrm{MR}$ \\
\hline $2 \times \times \times$ & $\mathbf{M} / \cdots$ & $(M R) \times\left(e . a .^{\prime}\right) \rightarrow M R$ \\
\hline $3 \times x \times$ & $\mathrm{S} / \cdots$ & $(M R)-\left(e . a .^{\prime}\right) \rightarrow M R$ \\
\hline $4 \times \times \times$ & $\mathrm{L} / \cdots$; & (e. a. $) \rightarrow M R$. \\
\hline $5 \times \times \times$ & $\mathrm{JP} / \cdots$ & Jump to e. a. if $(M R)>0$. \\
\hline $6 \times \times \times$ & $\mathrm{D} / \cdots$; & $(\mathrm{MR}) /\left(\mathrm{e} . \mathrm{a} .{ }^{\prime}\right) \rightarrow \mathrm{MR}$ \\
\hline $7 \times \times \times$ & $\mathrm{JM} / \cdots ;$ & Jump to e. a. if $(M R)<0$. \\
\hline $8 \times \times \times$ & & $\begin{array}{l}\text { Jump to the subroutine (the first } \\
\text { address is } \times \times \times \text { ) consisting of the } \\
\text { ordinary machine language. }\end{array}$ \\
\hline $9 \times \times x$ & $\mathbf{J Z} / \cdots$ & Jump to e. a. if $(M R)=0$. \\
\hline$A \times \times \times$ & $\mathbf{J} / \cdots ;$ & Jump to e. a. \\
\hline$B \times \times x$ & DI/A; & Trunc $\left((M R) /\left(\right.\right.$ e. a. $\left.\left.{ }^{\prime}\right)\right) \rightarrow M R$. \\
\hline$C \times \times \times$ & $\mathbf{J S} / \cdots$ & $\begin{array}{l}\text { Jump to the subroutine starting } \\
\text { from e. a. }\end{array}$ \\
\hline $\mathrm{D} \times \times \times$ & $\mathbf{T} / \cdots$ & $(M R) \rightarrow$ e. a.' \\
\hline $\mathrm{E} \times \times \times$ & $\operatorname{IMSK} / \cdots ;$ & $\begin{array}{l}\text { (e. a.) }+2 \rightarrow \text { e. a. and skip if the re- } \\
\text { sult is zero. Replace } 2 \text { with } 3 \text { in } \\
\text { MACRO/3. }\end{array}$ \\
\hline $\mathbf{F} \times x \times$ & IMS/ $/ \cdots ;$ & $\begin{array}{l}\text { (e. a.) }+1 \rightarrow \text { e. a. and skip if the re- } \\
\text { sult is zero. }\end{array}$ \\
\hline
\end{tabular}

(MR): The content of the pseudo main register MR (floating point number).

(e. a.'): The content of the 2 consecutive storages whose first address is the effective one (floating point number). Replace 2 by 3 in MACRO/3.

(e. a.): The content of the effective address (single length integer).

$(A)+(B) \rightarrow C$ : Add (B) to (A) and put into the storage C. 
Table 2. Pseudo Instructions $(8 \times \times \times)$

\begin{tabular}{|c|c|}
\hline $\begin{array}{l}\text { Mnemonic code } \\
\text { (in } \mathrm{ON} / 1)\end{array}$ & Operation \\
\hline ABS2/; & $|(\mathbf{M R})| \rightarrow \mathrm{MR}$ \\
\hline RTS2/; & Trunc (MR) $\rightarrow$ BK (a register of 1 word). \\
\hline FEED/; & Carriage-return and line-feed. \\
\hline $\left.\begin{array}{l}\text { PRI2/; } \\
\text { PRE2/; } \\
\text { PRF2/; }\end{array}\right\}$ & Print out (MR) by TTY with the format of I, E or F \\
\hline R2PT/; & Read a real number from PTR and store it into MR. \\
\hline \multicolumn{2}{|c|}{$\begin{array}{l}\text { ABS } 3 / ; \text { RTS } 3 / ; \text { FEED } / ; \text { PRIS } / ; \text { PRIL } / ; \text { PRES } / ; \text { PREL } / ; \text { PRFS } / ; \text { PRFL/; } \\
\text { and R3PT/; are the pseudo instructions in MACRO/3 and are similar to those in } \\
\text { MACRO/2 stated above. } \\
\text { Some of the subroutines called by above pseudo instructions are described in } \\
\text { Section } 7 .\end{array}$} \\
\hline \multicolumn{2}{|c|}{$\begin{array}{l}\text { Mnemonic code } \\
\text { (in } \mathrm{ON} / 1)\end{array}$} \\
\hline \multicolumn{2}{|r|}{$\sin (M R) \rightarrow M R$} \\
\hline \multicolumn{2}{|r|}{$\cos (\mathrm{MR}) \rightarrow \mathrm{MR}$} \\
\hline \multicolumn{2}{|r|}{$\arctan (\mathrm{MR}) \rightarrow \mathrm{MR}$} \\
\hline \multicolumn{2}{|r|}{$\exp (\mathrm{MR}) \rightarrow \mathrm{MR}$} \\
\hline \multicolumn{2}{|r|}{$\log _{e}(\mathrm{MR}) \rightarrow \mathrm{MR}$} \\
\hline \multicolumn{2}{|r|}{$\sqrt{(\mathbf{M R})} \rightarrow \mathbf{M R}$} \\
\hline \multicolumn{2}{|r|}{$(\mathrm{MR})^{(\mathrm{BK})} \rightarrow \mathrm{MR}^{*}$} \\
\hline \multicolumn{2}{|r|}{$(\mathrm{MR})^{\left(*+1^{\prime}\right)} \rightarrow \mathrm{MR}^{* *}$} \\
\hline
\end{tabular}

* Before using the pseudo instruction $A \uparrow N /$; we must store the exponent into BK. For this, we may use RTS2/; (or RTS3/;) which truncates (MR) into single length integer and store it into BK. For example, by the execution of the sequence of the pseudo instructions (L/A; RTS2/; (or RTS3/;) L/B;A A N/; T/C;), $\left(B^{\prime}\right)$ Trune $\left(A^{\prime}\right) \rightarrow C^{\prime}$ performs, where $\left(A^{\prime}\right)$ means the content of the 2 consecutive storages whose first address is $\mathbf{A}$ and Trunc ( $\mathbf{A}^{\prime}$ ) means to truncate the fractional part of $\left(A^{\prime}\right)$.

** $\left(*+1^{\prime}\right)$ means the content of the 2 consecutive storages next to the storage in which $A \uparrow X /$; is stored. Before using the pseudo instruction $\mathrm{A} \uparrow \mathrm{X} /$; we must store the exponent into the storage which consists of 2 (or 3 ) consecutive storages next to the pseudo instruction. For example, by the execution of the sequence of the pseudo instructions (L/A; T/*+3; L/B; A $\uparrow \mathrm{X} / ; ; ;$ (;) $\mathrm{T} / \mathrm{C} ;$ ), $\left(B^{\prime}\right)^{\left(A^{\prime}\right)} \rightarrow C^{\prime}$ is performed. 
The pseudo instructions must be used between the two particular instructions MCR2/; (or MCR3/;) and $\mathrm{H} /$; . When the source program is assembled by $\mathrm{ON} / 1$, the instructions and the pseudo instructions are translated to the corresponding machine codes, and the object program is completed. When the object program is executed, the particular instruction MCR2/; (or MCR3/;) (for example, in the address $M$ ) works to store the address $M+1$ into the first location of the interpreter MACRO/2 (or MACRO/3) and transfer the control to the second location of it. So MACRO/2 (or MACRO/3) begins to operate and the next pseudo instruction is interpreted and the corresponding operation is performed, and so on. Once the pseudo instruction $\mathrm{H} /$; (for example, in the address $\mathrm{A}$ ) is interpreted, the interpretation by MACRO/2 (or MACRO/3) ends, and the instruction in the address $A+1$ and the following are performed as the "ordinary" instructions.

Because the mnemonic codes of the pseudo instructions are similar to those of the instructions of ON/1, MACRO/2 and MACRO/3 are very useful for the computations using the floating numbers, and play an important rôle to extend the functions of our computer MINI.

\section{Main flow of MACRO/2 (or MACRO/3)}

The main flow of the MACRO/2 (or MACRO/3) is given in Fig. 4.

\section{Subroutines for arithmetic operations}

The general flow in the arithmetic operations (addition, subtraction, multiplication and division) is as follows.

(1) The data is transferred from the effective address to SR,

(2) The arithmetic operation is done using the contents in MR and SR,

(3) The result is stored into MR.

To do this, we develop the subroutines OPER, ARL, SRL, MRL, DRL, DI and MVSM.

\subsection{Subroutine OPER.}

In OPER the following are done.

(1) The data is transferred from the effective address degignated by (EA) to SR,

(2) According to the content of OP, the operation is performed by the corresponding subroutine.

\subsection{Subroutines ARL AND SRL.}

ARL is the subroutine which adds the data in SR to MR and stores the result 

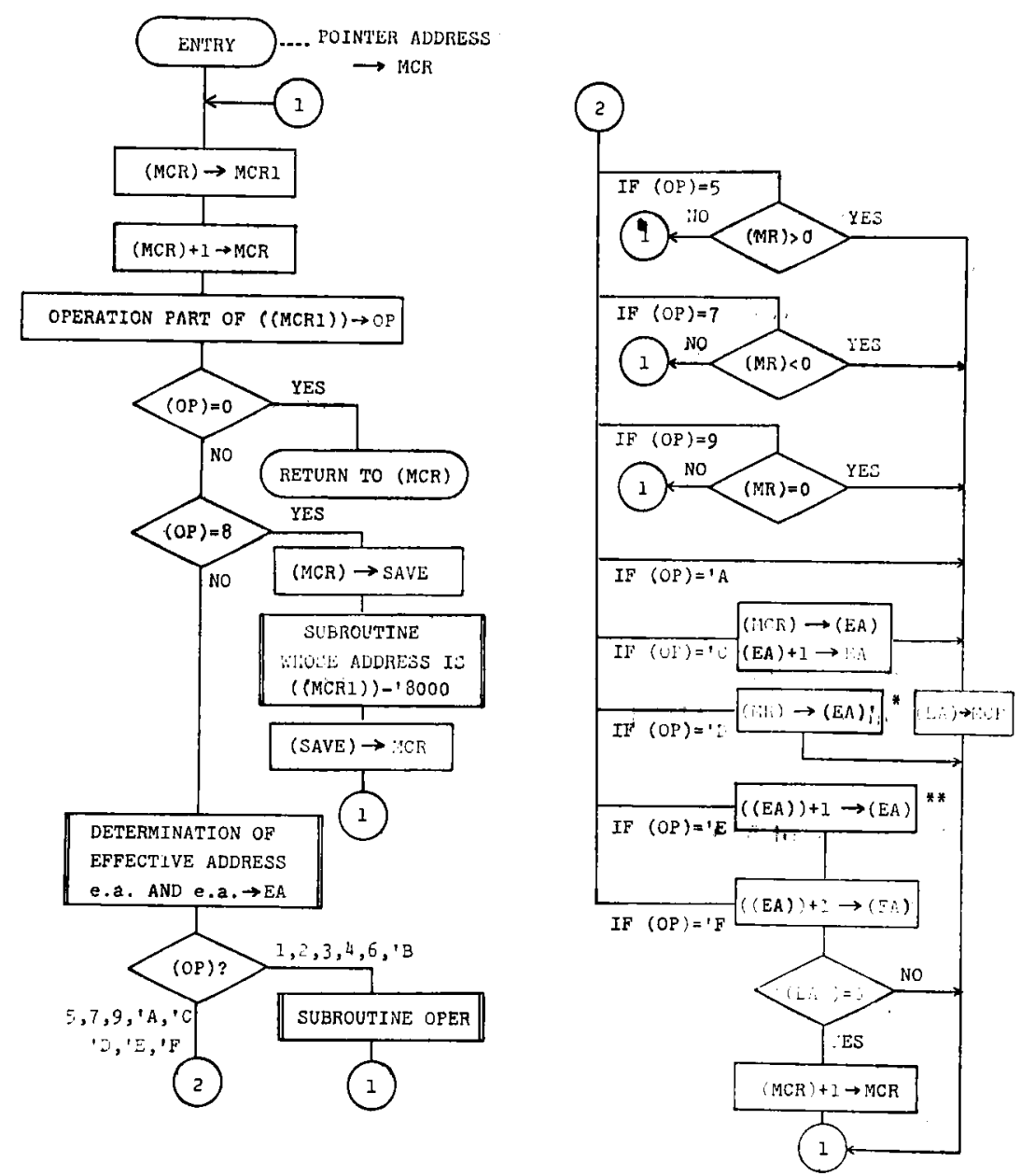

Fig. 4. Main Flow of the MACRO/2 (or MACRO/3).

* (EA) means the content of EA, that is, e.a. (EA)' is e.a.'.

** Replace $((\mathrm{EA}))+1$ by $((\mathrm{EA}))+2$ in MACRO/3.

into MR. That is $(M R)+(S R) \rightarrow M R$. The method is conventional and the details are omitted. The subroutines used in ARL are as follows.

Subroutine

\section{Explanation}

MVSM

SRSR

SRMR

CMMR
$(\mathrm{SR}) \rightarrow \mathrm{MR}$

Shift the content of the mantissa of SR to the right by 1 bit and adds 1 to SR3.

Shift the content of the mantissa of MR to the right by 1 bit and adds 1 to MR3.

Two's complement of (the mantissa of MR) $\rightarrow$ the mantissa of MR. 
AMS (mantissa of MR) $+($ mantissa of SR) $\rightarrow$ the mantissa of MR.

NOR Normalize (MR) so that the 14th bit of MRI becornes 1 .

In $S R L$ we do $(M R)-(S R) \rightarrow M R$, that is, the sign of $(S R)$ is changed and ARL is called.

\subsection{Subroutine MRL.}

In MRL we do (MR) $\times(S R) \rightarrow M R$. This is done by the method "Shift MR and SR to the left". The subroutines used in MRL are SLMR, AMS and NOR.

Subroutine

SLMR

SLWR

\section{Explanation}

Shift (the mantissa of MR) to the left by 1 bit. Shift (WR) to the left by 1 bit.

\subsection{Subroutine DRL.}

In DRL we do (MR)/(SR) $\rightarrow$ MR, and the content of MR is not varied if (SR) $=0$. This is done by the method "Shift MR and SR to the right". The subroutines used in DRL are SRMR, SLMR, SMS, AMS and NOR. The work of SMS is (the mantissa of MR) - (the mantissa of SR) $\rightarrow$ the mantissa of MR.

\subsection{SUbRoutine DI.}

It needs often to omit fractions of the resultant of DRL, and DI is developed. Thus DRL is called in DI, the fractional part is cut off and then the content of MR becomes an integer. The subroutines used in DI are DRL, RTI and NOR. The work of RTI is to cut off the fractional part in (MR).

\section{Subroutines used for reading and writing a number}

\subsection{Subrotine RRL.}

This is developed to read a number with type I, F or E by PTR and store it into MR as a normalized floating binary number. The subroutines used in RRL are READ, D09 and CTR.

In READ we can read a character represented by ASCII code into the accumulator of MINI. D09 is the subroutine which separates the characters $0 \sim 9$ from others. CTR is very important, and changes a code obtained by READ into a real number if the code is one of $0 \sim 9$, or else not changes.

The subroutines used in CTR are MR10 and AMS. We do (the mantissa of 
MR) $\times 10 \rightarrow$ the mantissa of MR using MR10, in which SRMR and AMS are used.

\subsection{SUBROUTINES USED FOR WRITING A NUMBER}

The subroutines used for writing a number in MR are as follows.

\begin{tabular}{|c|c|}
\hline Subroutine & Format \\
\hline PR12 & $\Perp_{Z Z Z Z Z Z Z X}$ \\
\hline PRF2 & $=X \underbrace{X \cdot X \cdot X \cdots X}_{7 \text { Figures }}$ \\
\hline PRE2 & 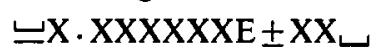 \\
\hline
\end{tabular}

$X$ means a decimal number of 1 figure, $Z$ means $X$ if the degit is not zero, or else zero suppression, $=$ means a space or a minus sign, and \pm means a puls or minus sign.

In MACRO/3 we have the following subroutines to write a number in MR.

\begin{tabular}{|c|c|}
\hline Subroutine & Format \\
\hline PRIS & $=Z Z Z Z Z Z Z X_{\Perp}$ \\
\hline PRIL & 凹ZZZZZZZZZZZX \\
\hline PRFS & $=\mathrm{X} \cdot \underbrace{\ldots \mathrm{X} \cdot \mathrm{X} \cdots \mathrm{X}}_{7 \text { Figures }}$ \\
\hline PRFL & $=X \underbrace{X X X}_{12 \text { Figures }}$ \\
\hline PRES & $\unlhd X \cdot X X X X X X E \pm X X_{\sqcup}$ \\
\hline PREL & $=X \cdot X X X X X X X X X X X E \pm X X$ \\
\hline
\end{tabular}

In these subroutines we used the pseudo instructions of MACRO/2 (MACRO/ 3). The detail are omitted.

\section{Elementary functions ${ }^{1 / 2)}$}

The values of sine, cosine and arctangent functions are evaluated by means of the suitable polynomials. The values of exponential and logarithmic functions are evaluated by means of the rational functions. The square root is evaluated by Newton's method.

The value of $A^{B}$ is computed by multiplying 1 by $A B$ times if $B$ is a positive integer, and by replacing $A^{B}$ with $1 / A^{|B|}$ if $B$ is a negative integer. And the value 1 is adopted for $A^{B}$ if $B=0$. If $B$ is not a integer, $A^{B}$ is computed by means of $\exp (B \times \ln (A))$. The full detail are omitted. 


\section{References}

[1] T. Simauti, Approximation Formulas for Some Elementary Functions, Comm. Math. Univ. St. Pauli, XII (1963), 23-35.

[2] E. W. Cheney, Introduction to Approximation Theory, Mcgraw-Hill, (1966). 